\title{
Automatic Estimation of Error in Voxel-Based Registration
}

\author{
William R. Crum, Lewis D. Griffin, and David J. Hawkes \\ Division of Imaging Sciences, Thomas Guy House, Guy's Hospital, Kings College London, \\ London SE1 9RT, UK \\ \{bill.crum, lewis.griffin, david.hawkes\}@kcl.ac.uk
}

\begin{abstract}
Image registration driven by similarity measures that are simple functions of voxel intensities is now widely used in medical applications. Validation of registration in general remains an unsolved problem; measurement of registration error usually requires manual intervention. This paper presents a general framework for automatically estimating the scale of spatial registration error. The error is estimated from a statistical analysis of the scale-space of a residual image constructed with the same assumptions used to choose the image similarity measure. The analysis identifies the most significant scale of voxel clusters in the residual image for a coarse estimate of error. A partial volume correction is then applied to estimate finer and sub-voxel displacements. We describe the algorithm and present the results of an evaluation on rigid-body registrations where the ground-truth error is known. Automated measures may ultimately provide a useful estimate of the scale of registration error.
\end{abstract}

\section{Introduction}

Image registration is widely used in medical analysis with continuing efforts to improve, develop and validate existing algorithms [1]. A convenient distinction can be made between "manual" registration algorithms which use identified features and "automatic" algorithms that use voxel intensities. While the manual methods have some inherent error estimation through the identification of corresponding features, the automatic methods can only check that the voxel similarity measure is increasing and require further input (such as the laborious and error-prone identification of landmarks e.g. [2]) to measure registration error on a case-by-case basis. Where automatic registration algorithms are employed in large studies their behaviour is usually characterised on a representative subset of data.

In this paper we propose an approach for automatic estimation of error in voxelbased registration that combines statistical tests of significance on a residual image with a scale-selection process. The target application is retrospective estimation of registration error but it is also related to the problem of detection of change since image differences post-registration may represent biological changes of interest rather than error (e.g. lesion development in Multiple Sclerosis or contrast enhancement over time). In this latter case our approach will assign a spatial scale to such features and further interpretation will be required. 


\section{Method}

A natural framework for examining image structures at different scales is provided by scale-space theory [3]. Images can be analysed at any scale desired by convolving them with a Gaussian filter of a specified standard deviation (s.d.). In image registration, it is common to examine a digital subtraction image (or more generally a residual image) post-registration to identify error or residual image differences. Our general approach is to identify the most significant scale associated with a structure in the residual image and interpret this as the scale of registration error. There are three required steps. First, a suitable residual image must be constructed, then scale selection must be applied to significant voxel clusters and finally these voxel intensities must be transformed into estimates of spatial displacement.

\subsection{Constructing the Residual Image}

The simplest case is mono-modal images assumed to differ only by additive Gaussian noise that are often registered by minimising their r.m.s. intensity difference. For multi-modal registration there is generally not a one-to-one relationship between the intensity at corresponding points in the two images. This can be dealt with by constructing a simulated difference image from the joint intensity probability distribution of the image pair (the target and source). For each voxel intensity in the source image, the most probable intensity in the target is selected and subtracted from the actual target voxel intensity. For true multi-modal images that do not have a one-to-one intensity relationship between source and target, the most probable intensity will not necessarily be the correct choice and will be a source of error.

\subsection{Establishing Approximate Scale of Image Differences}

Fig. 1(a) shows a 1D intensity profile of a displacing block edge, together with the 1D subtraction profile showing high signal over the region where the edge has displaced. Fig. 1(b) shows how the response of the difference profile to a Gaussian filter, once normalised by the noise response to the filter, exhibits a peak at a specific scale, $\sigma$. This suggests that by convolving the residual image with Gaussian filters of different scales to construct an isotropic scale-space and then normalising for noise, statistical tests on the intensity at each voxel can be used to find the most significant scale for features in the residual image.

In scale-space images there is substantial correlation between the intensities of neighbouring voxels introduced by the filtering process and the commonly used Bonferroni correction for large numbers of statistical tests is overly conservative. This problem has been addressed by Worsley et al [4] using the theory of Gaussian random fields to set the threshold for statistical significance from an estimate of the smoothness of the image and shape and extent of the search volume. The significance level sets the rate of false positive voxel clusters. We adopt this approach here assuming stationary Gaussian noise. 
(a)
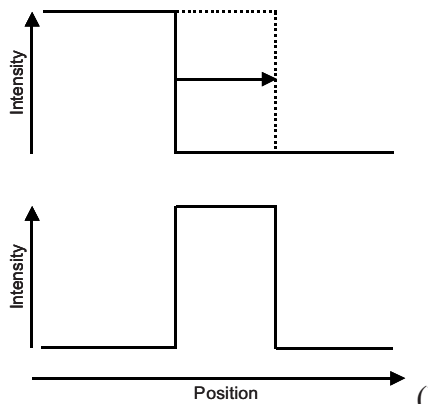

(b)

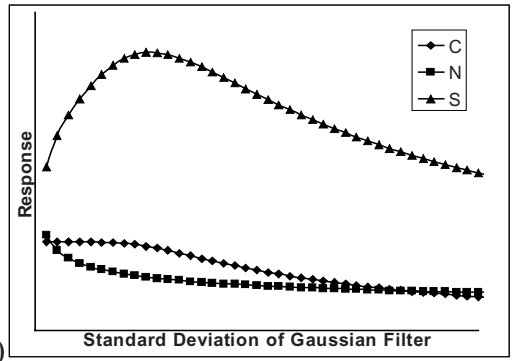

Fig. 1. 1D Edge Displacement. (a) Intensity profiles of a displacing edge and the difference between the displaced and the undisplaced edge. (b) Schematic showing the effect of convolving a Gaussian filter with the difference profile. $\mathrm{C}=$ Convolution of difference profile, $\mathrm{N}=$ Convolution of Gaussian noise, $\mathrm{S}=\mathrm{C} / \mathrm{N}$ is the normalised response of the difference profile

\subsection{Measuring Sub-voxel Displacements}

The method described in section 2.2 results in a relatively coarse assignment of scale that will be insensitive to sub-voxel edge-displacements. This is because tests are voxel-wise and a range of sub-voxel displacements may all be assigned the scale of the same applied Gaussian filter due to partial volume effects. To distinguish these displacements a single-voxel partial volume model of edge-displacement is used. In Fig. 2(a) an edge intensity profile is shown in different positions within the central voxel in the target (top) and source (bottom) images. The intensities of neighbouring voxels are presumed identical and equal to $I_{1}$ (right of centre) and $I_{2}$ (left of centre). The intensity of the central voxel in each image is related to the position of the edge (denoted by $\alpha$ and $\beta$ ) and given by:

$$
T_{C}=\alpha I_{2}+(1-\alpha) I_{1}, \quad S_{C}=\beta I_{2}+(1-\beta) I_{1}
$$

where $T_{C}$ is the central intensity in the target image and $S_{C}$ is the central intensity in the source image. In Fig. 2(b) the intensity profile in the subtraction image is shown together with the intensities of the voxels in the subtraction image. Then the central voxel intensity in the difference image, $D_{C}$, is given by:

$$
D_{C}=T_{C}-S_{C}=(\alpha-\beta)\left(I_{2}-I_{1}\right)
$$

Writing $\Delta=-(\alpha-\beta)$ it can be seen that $\Delta=-D_{C} /\left(I_{2}-I_{1}\right)$. Therefore the edge displacement can be estimated from the voxel intensity in the difference image normalised by the intensity gradient across the voxel in either the target or source image. In practice the target and residual images are resampled to the most significant scale so that the edge displacement can be modelled as within-voxel. 
(a)
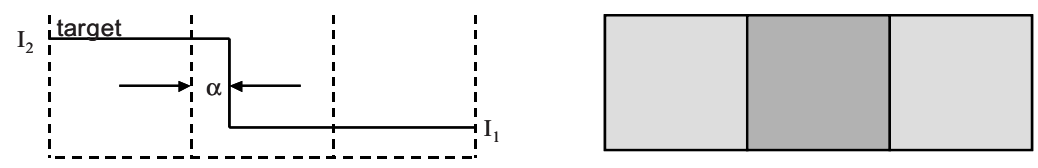

(b)

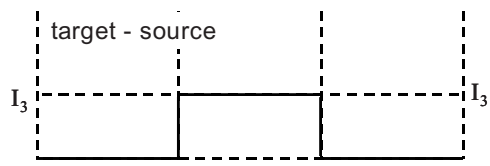

Fig. 2. The partial-volume model of a displacing edge. (b) The intensity profile of an edge within a voxel in two images. (b) The difference image and difference profile

\section{Experiments}

The algorithm was implemented in GNU C/C++ under Redhat Linux. Gaussian filtering was accomplished by multiplication in the frequency domain. Run times were < 5 minutes per case for the full algorithm applied to 2D images.

(a)
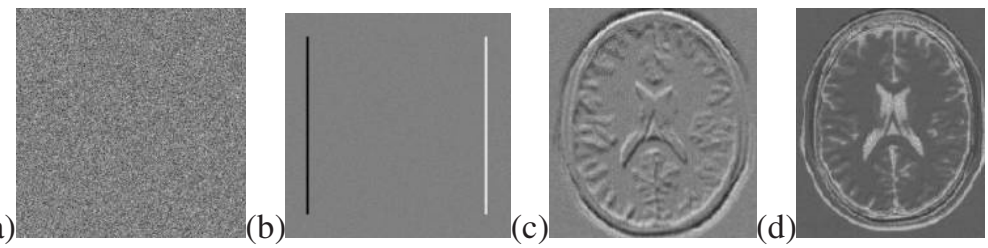

Fig. 3. Residual images used in the experiments (a) noise field (b) displaced square (c) T1-brain slices (d) T2 and T1 brain slices. (a)-(c) are difference images and (d) is a residual image computed from the joint intensity probability distribution. See text for further details

\subsection{Rate of False-Positives}

We need to check that the thresholds for significance are being set correctly so that only the expected number of false-positive significant voxel clusters are detected. Two independent 512x512 Gaussian noise images (Fig. 3(a)) were created and the number of significant voxel clusters determined over eleven scales in the range $[0.8,15]$ pixels and significance levels varying between $[0.005,0.5]$. The experiment was repeated 1000 times for clusters detected as described in Section 2.1.

\subsection{Detecting a Displacing Edge}

Two copies of an image of a constant intensity square were created and one was displaced by varying amounts along the $x$-axis (one case is shown in Fig. 3(b)). Noise was added to each image and the displacement was estimated by (a) finding the mean most significant scale of difference and (b) the mean most significant scale after ap- 
plying the partial volume correction. The experiment was run for 9 sub-voxel displacements in the range $[0.1,0.9]$ and 8 voxel displacements in the range $[1,10]$. The experiment was repeated for 10 different levels of noise in the images in the range $[1 \%, 10 \%]$. Here and in the following experiments the significance level was set equal to 0.01 at all scales.

\subsection{Detecting 2D Rigid-Body Motion from Subtraction Images}

Two copies of a single slice of the MNI BrainWeb T1-weighted digital phantom were used [5], [6]. One copy was displaced by a random amount in the range $[0,4 \sqrt{2}]$ voxels (i.e. up to \pm 4 voxels in each of the $x$ and $y$ directions) and rotated by a random amount in the range $\pm 2^{\circ}$ forty times (Fig. 3(c)). Independent Gaussian noise of mean $3 \%$ was added to each image before analysis. The images were ranked for misregistration by computing the mean voxel-displacement in brain in each case. The mean displacement between the displaced image and the static image was then estimated as in 3.2 (b) for each of the forty cases.

\subsection{Detecting 2D Rigid-Body Motion from Residual Images}

Experiment 3.3 was repeated using the statistical residual image (Fig. 3(d)). In the first case the same pairs of T1-weighted images were used and in the second case the static image was replaced by a T2-weighted image.

\section{Results}

\subsection{Rate of False Positives}

Fig. 4 shows the total number of false positive significant voxel clusters detected over all scales compared with the expected number for the range of significance levels.

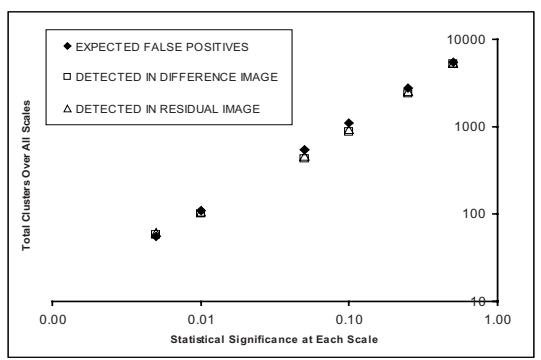

Fig. 4. The number of false positive voxel clusters detected in a Gaussian random noise field as a function of the statistical significance level 


\subsection{Detecting a Displacing Edge}

Fig. 5 shows the most significant mean recovered scale of displacement as a function of the applied displacement for each noise level for (a) the simple approach and (b) the partial volume correction. Note that this mean is only computed over voxels that are significant in the residual image. There is a highly significant linear trend for all noise levels in the partial volume graph that extends to sub-voxel applied displacements.
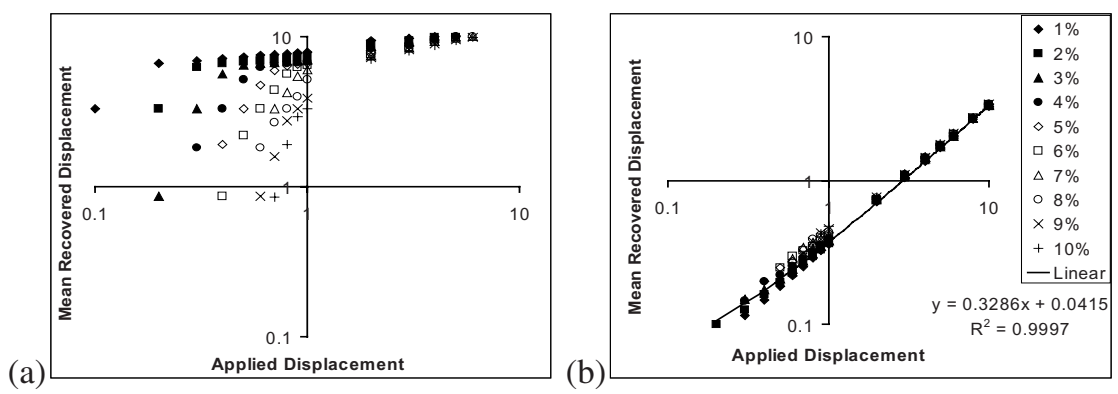

Fig. 5. The recovered mean displacement as a function of applied mean displacement for a translating square subject to 10 different noise levels (a) the most significant scale (b) with partial volume correction. The trend line is constructed for the $1 \%$ noise level
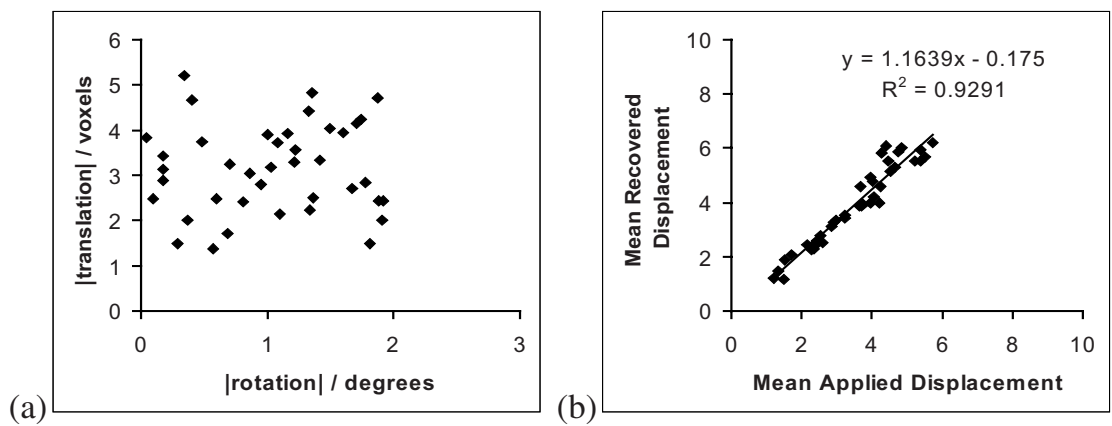

Fig. 6. (a) The distribution of $2 \mathrm{D}$ random translations and rotations applied to the example brain data. (b) The relationship between the mean applied voxel displacement and the mean recovered voxel displacement recovered the subtracted pairs of T1-weighted brain scans.

\subsection{Detecting 2D Rigid Body Motion from Subtraction Images}

Fig. 6 (a) shows the distribution of applied rotations and translations and (b) the relationship between the magnitude of the applied global displacement and the magnitude of the mean recovered displacement for the forty test cases. 


\subsection{Detecting 2D Rigid Body Motion from Residual Images}

Fig. 7 shows the results for the same test cases as in figure 6 but with the displacement recovered from a statistical residual image rather than a difference image. The graphs show (a) T1-T1 displacement and (b) T2-T1 displacement.
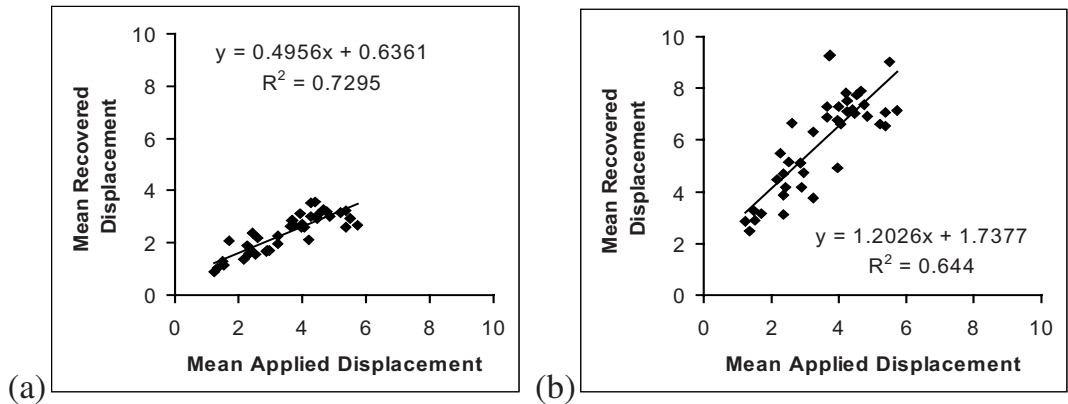

Fig. 7. The relationship between mean applied and mean recovered voxel displacement for the cases shown in figure 6 calculated from (a) T1-T1 statistical residual image (b) T2-T1 statistical residual image

\section{Discussion}

We propose that registration error can be estimated from a post-registration residual image and have shown that rigid-body mis-registrations can be ranked by mean voxel displacement with a linear relationship between estimated and known mean voxel displacement. The techniques in this paper generalise immediately to 3D data. However, we have taken a simple approach to address a complex problem and a more general registration scenario will require further innovation. In particular, our attempt to treat the multi-modal case is simplistic and the results are data-dependent (Fig. 7). Spatial localisation of estimated error is the ultimate goal but will require a more thorough analysis. There is a deep relationship between the size and configuration of corresponding objects in the source and target images and the structures seen in residual images that should be explored and exploited. The displacement of objects that do not overlap will not be recovered with the current naïve approach.

There are other caveats. First, for simplicity we have applied the same statistical significance at all scales; this implies repeated tests at each voxel. Our experience is that the results are relatively insensitive to the significance level but note that previous work [9] suggests a more principled way of searching for significant structure within a scale-space. It may be more appropriate to specify the total significance across all scales and to weight the significance to favour smaller scale structures. Second, the structure in the residual image must be interpreted in light of the applied transformation model. A structure-less residual image may result from a highly non-physical transformation that does not reflect true correspondence. The particular transformation model will also affect spatial correlation of features in the residual image and this might be exploited to differentiate mis-registration from true change; for instance a rigid-body transformation will introduce correlated structures at opposing faces of a 
displaced object. In the future a scale-selective feature analysis of both the target image and the residual image may enable tighter bounds on error to be found.

These methods will not replace detailed evaluation studies (e.g. [7], [8]) but will complement them with estimates that can be obtained retrospectively or prospectively without special provision. The principle that an analysis of the residual image structure can be used to estimate the likely registration error or to automatically compare attempts at registration is a powerful one. With automatic voxel-based registration increasingly applied in large studies, novel methods to automatically monitor and estimate registration error are urgently required. Such methods will assist in validation and monitoring of existing techniques and ultimately may be used to initialise or to drive registration.

Acknowledgments. The authors are grateful for the intellectual and financial support of the Medical Images and Signals IRC (EPSRC and MRC GR/N14248/01).

\section{References}

1. Hajnal, J.V., Hill D.L.G. and Hawkes, D.J.: Medical Image Registration, CRC Press, Boca Raton, USA (2001)

2. Grachev, I.D., Berdichevsky D., Rauch, S.L., Heckers, S., Kennedy, D.N., Caviness V.S. amd Alpert, N.M.: A Method For Assessing the Accuracy of Intersubject Registration of the Human Brain Using Anatomic Landmarks, NeuroImage 9 (1999) 250-268

3. Koenderink, J.J.: The Structure of Images. Biological Cybernetics 50(5) (1984) 363-370

4. Worsley, K.J., Marrett, S. P., Neelin, Vandal, A.C., Friston, K.J. and Evans, A.C. : A Unified Statistical Approach for Determining Significant Signals in Images of Cerebral Activation. Human Brain Mapping 4 (1996) 58-73

5. Collins, D.L., Zijdenbos, A.P., Kollokian, V., Sled, J.G., Kabani, N.J., Holmes C.J. and Evans, A.C.: Design and Construction of a Realistic Digital Brain Phantom. IEEE Transactions on Medical Imaging 17(3) (1998) 463-468

6. Kwan, R.K.-S., Evans, A.C. and Pike, G.B.: MRI Simulation-Based Evaluation of Image Processing and Classification Methods. IEEE Transactions on Medical Imaging 18(11) (1999) 1085-1097

7. Hellier, P., Barillot, I., Corouge, B., Gibaud, G., Le Goualher, G., Collins, D.L., Evans, A., Malandain, G., Ayache, N., Christensen, G.E. and Johnson H.J.: Retrospective Evaluation of Intersubject Brain Registration. IEEE Transactions on Medical Imaging, 22(9) (2003) 11201130

8. Grachev, I.D., Berdichevsky D., Rauch, S.L., Heckers, S., Kennedy, D.N., Caviness V.S. amd Alpert, N.M.: A Method For Assessing the Accuracy of Intersubject Registration of the Human Brain Using Anatomic Landmarks, NeuroImage 9 (1999) 250-268

9. Worsley, K.J., Marrett, S., Neelin, P. and Evans, A.C.: Searching Scale Space for Activation in PET Images. Human Brain Mapping 4 (1996) 74-90 